\title{
Cyclic deformation of nanocrystalline and ultrafine-grained nickel
}

\author{
S. Cheng ${ }^{\text {a,b,*, }}$ J. Xie ${ }^{\text {c }}$, A.D. Stoica ${ }^{\text {b }}$, X.-L. Wang ${ }^{\text {b }}$, J.A. Horton ${ }^{\text {d }}$, D.W. Brown ${ }^{\text {e }}$, \\ H. Choo ${ }^{\text {a,d }}$, P.K. Liaw ${ }^{\mathrm{a}}$ \\ a Department of Materials Science and Engineering, The University of Tennessee, Knoxville, TN 37996, USA \\ ${ }^{\mathrm{b}}$ Neutron Scattering Science Division, Oak Ridge National Laboratory, Oak Ridge, TN 37831, USA \\ ' State Key Laboratory of Nonlinear Mechanics, Institute of Mechanics, Chinese Academy of Sciences, Beijing 100190, China \\ ${ }^{\mathrm{d}}$ Materials Science and Technology Division, Oak Ridge National Laboratory, Oak Ridge, TN 37831, USA \\ ${ }^{\mathrm{e}}$ Los Alamos Neutron Science Center, Los Alamos National Laboratory, Los Alamos, NM 87545, USA
}

Received 4 September 2008; received in revised form 28 October 2008; accepted 5 November 2008

Available online 26 December 2008

\begin{abstract}
The cyclic deformation behavior of ultrafine-grained (UFG) Ni samples synthesized by the electrodeposition method was studied. Different from those made by severely plastic deformation, the UFG samples used in this study are characterized by large-angle grain boundaries. Behaviors from nanocrystalline Ni and coarse-grained Ni samples were compared with that of ultrafine-grained Ni. With in situ neutron diffraction, unusual evolutions of residual lattice strains as well as cyclic hardening and softening behavior were demonstrated during the cyclic deformation. The microstructural changes investigated by TEM are discussed with respect to the unusual lattice strain and cyclic hardening/softening.
\end{abstract}

(C) 2008 Acta Materialia Inc. Published by Elsevier Ltd. All rights reserved.

Keywords: Ultrafine-grained; Cyclic deformation; Neutron diffraction; Cyclic hardening/softening; Lattice strain

\section{Introduction}

With in-depth investigations of the plastic deformation of nanocrystalline $(\mathrm{NC})$ metals in recent years, many distinctive deformation behaviors were reported for these materials, and accordingly new deformation mechanisms were proposed [1-7]. For example, under certain deformation conditions, $\mathrm{NC} \mathrm{Ni}$ could deform by partial dislocations and twinning $[8,9]$. Moreover, in situ X-ray diffraction studies demonstrated fully reversible peak broadening in $\mathrm{NC} \mathrm{Ni}$ during tensile loading/unloading, suggesting that dislocation debris might not be left over upon unloading [10]. Considerable efforts were also devoted to the deformation

\footnotetext{
${ }^{*}$ Corresponding author. Address: Department of Materials Science and Engineering, The University of Tennessee, Knoxville, TN 37996, USA. Tel.: +1 865974 2683; fax: +1 8659744115 .

E-mail address: scheng1@utk.edu (S. Cheng).
}

mechanism investigations of ultrafine-grained (UFG) metals. In situ X-ray diffraction showed irreversible peak broadening after unloading in UFG $\mathrm{Ni}$, indicating that the deformation resulted in a build-up of dislocation networks, as in conventional coarse-grained (CG) metals [11]. However, our recent in situ synchrotron X-ray diffraction studies suggest that, in addition to dislocation activities, deformation twinning could also play a significant role in the tensile deformation of UFG Ni [12]. Thus, as in NC Ni, the deformation mechanisms in UFG are also complex and not well understood. Furthermore, cyclic deformation of $\mathrm{NC}$ and UFG metals has been shown to be very different from their CG counterparts [13-20]. However, so far, only limited investigations were carried out on cyclic deformation of UFG metals, and most of them were focused on the fatigue response and damage characterization. The underlying deformation mechanism was rarely addressed.

One the other hand, to date, the UFG metals studied were mostly produced by severely plastic deformation (SPD) 
method, e.g., equal-channel angular pressing (ECAP) or rolling, etc. The microstructures of those UFG metals are usually very different from those of well annealed samples. Firstly, the grain boundaries of the UFG metals by SPD are often of small-angle type and non-equilibrium, far from the stable large-angle grain boundaries usually known in the polycrystalline materials $[21,22]$. Secondly, the grains are usually filled with pre-existing defects (e.g., high density of dislocations) [23-25]. Thus, the deformation behaviors reported in the literature are quite different from the samples with large-angle grain boundaries. In recent years, electrodeposition has found its way of making metals and alloys with very fine grain size [26]. To compare the deformation of UFG samples with that of conventional alloys, it is a prerequisite to have large-angle grain boundaries in UFG metals. In this study, we study the cyclic deformation behavior of electrodeposited UFG Ni with large-angle grain boundaries. With in situ neutron diffraction, the lattice strain and the macroscopic strain evolutions are investigated during cyclic deformation. We also compare the samples with different grain sizes. To understand the deformation mechanism, we characterize the microstructure changes from the as-received samples to the deformed samples by detailed transmission electron microscopy (TEM) observations. Combining the TEM observations with the evolutions of both the residual lattice strains and the macroscopic strain, the deformation pathways of UFG Ni were identified, and their role will be discussed with regard to the strain evolutions. With these model materials mimicking the structure of typical polycrystalline metals, we aim to clarify the intrinsic mechanism of cyclic deformation for this emerging group of materials.

\section{Experimental}

Nickel samples with four different grades of grain sizes, which cover a full range from nanocrystalline to the conventional coarse-grain regime, were used for comparison. The NC and UFG Ni samples were synthesized by a pulsed electrodeposition technique at Integran Technologies Inc. (Canada). The dimensions of the as-received $\mathrm{NC}$ and UFG Ni sheets were approximately $75 \mathrm{~mm} \times 75 \mathrm{~mm}$ in planar directions, and $\sim 1 \mathrm{~mm}$ in thickness. Flat dog-bone shaped pin-load samples were fabricated using an electrical discharge machine. The overall length of a fatigue sample was $75 \mathrm{~mm}$, with the gage length of $28 \mathrm{~mm}$, gage length of $4 \mathrm{~mm}$, and $0.6 \mathrm{~mm}$ in thickness. CG Ni foils with commercial purity were purchased from Alfa Aesar. Since the as-received CG Ni was cold rolled, recrystallization annealing was preformed at $450^{\circ} \mathrm{C}$ for $3 \mathrm{~h}$ [27]. In situ neutron diffraction with a time-of-flight method was conducted using SMARTS hydraulic load-frame at Los Alamos Neutron Science Center. The loading axis on the samples was at $45^{\circ}$ angle to the incident neutron beam, and the two detector banks were at $\pm 90^{\circ}$ to the incident beam. With this setting, the lattice strains in the loading direction and the transverse direction were determined using the $\pm 90^{\circ}$ detector banks, respectively. The lattice strains, defined as the relative lattice spacing change, i.e., $d^{h k l}-d_{0}^{h k l} / d_{0}^{h k l}$, are calculated from the peak position shift, where $d^{h k l}$ and $d_{0}^{h k l}$ are the $d$-spacings at the applied load and the reference load, respectively. Single-peak profile fitting was carried out using the general structure analysis systems (GSAS) software.

The cyclic deformation was conducted with a load-controlled mode, with the maximum stress, $\sigma_{\max }$ and the minimum stress, $\sigma_{\min }$ both maintained at constant levels. The cyclic loading was conducted at a frequency of $1 \mathrm{~Hz}$. To ensure a plastic deformation, all the samples were loaded with the $\sigma_{\max }$ greater than the yield stress $\left(\sigma_{0.2}\right)$, see Table 1 for details. The macroscopic strain evolution during cyclic loading was recorded using a strain extensometer clipped on the sample gage section. The grain misorientations and texture of the as-received materials were measured by electron backscattering diffraction (EBSD). The microstructures of the as-received samples and deformed samples were examined by TEM. The TEM specimens were prepared by twin-jet electropolisher using an etching solution of $25 \%$ nitric acid plus $75 \%$ methanol at $-45^{\circ} \mathrm{C}$. The TEM observation was performed using a Hitachi800 microscope operated at $200 \mathrm{kV}$.

\section{Results and discussions}

The microstructures as well as the grain size histograms of the as-received electrodeposited $\mathrm{Ni}$ are shown in Fig. $1 \mathrm{a}-\mathrm{c}$. The average grain size of the $\mathrm{NC} \mathrm{Ni}$ is around $20 \mathrm{~nm}$. For the Ni sample with a nominal grain size of $100 \mathrm{~nm}$ (Ni100), the grains have a rather broad grain size distribution, ranging from tens of $\mathrm{nm}$ to $1200 \mathrm{~nm}$. For the UFG Ni sample with a nominal grain size of $1000 \mathrm{~nm}$ (Ni1000), the grains range from $300 \mathrm{~nm}$ to $2 \mu \mathrm{m}$, but most of them are around $700-900 \mathrm{~nm}$. The grain boundaries of the UFG Ni are mostly clear and sharp, indicative of large-angle type.

Fig. 2 shows the grain-misorientation distributions of the as-received UFG $\mathrm{Ni}$ samples (both Ni100 and Ni1000) measured by EBSD. One can see that the grains are mostly orientated with large-angle boundaries in both Ni samples, as it is normally considered as a large-angle boundary when the misorientation is greater than $15^{\circ}$ [28]. Particularly, a predominant majority of misorientations appears at the angle of $\sim 60^{\circ}$. Note that the misorientations exhibited in our UFG samples are significantly superior to those made by SPD methods, in which the grain orientations were mostly below $15^{\circ}$ despite the grain size

Table 1

Parameters used in the in situ cyclic loading.

\begin{tabular}{llll}
\hline Samples & $\sigma_{\min }(\mathrm{MPa})$ & $\sigma_{\max }(\mathrm{MPa})$ & $\sigma_{0.2}(\mathrm{MPa})$ \\
\hline$d=20 \mathrm{~nm}$ & 50 & 850 & $\sim 820$ \\
$d=100 \mathrm{~nm}$ & 50 & 620 & $\sim 510$ \\
$d=1000 \mathrm{~nm}$ & 50 & $460 / 560$ & $\sim 450$ \\
CG Ni & 50 & 575 & $\sim 550$ \\
\hline
\end{tabular}



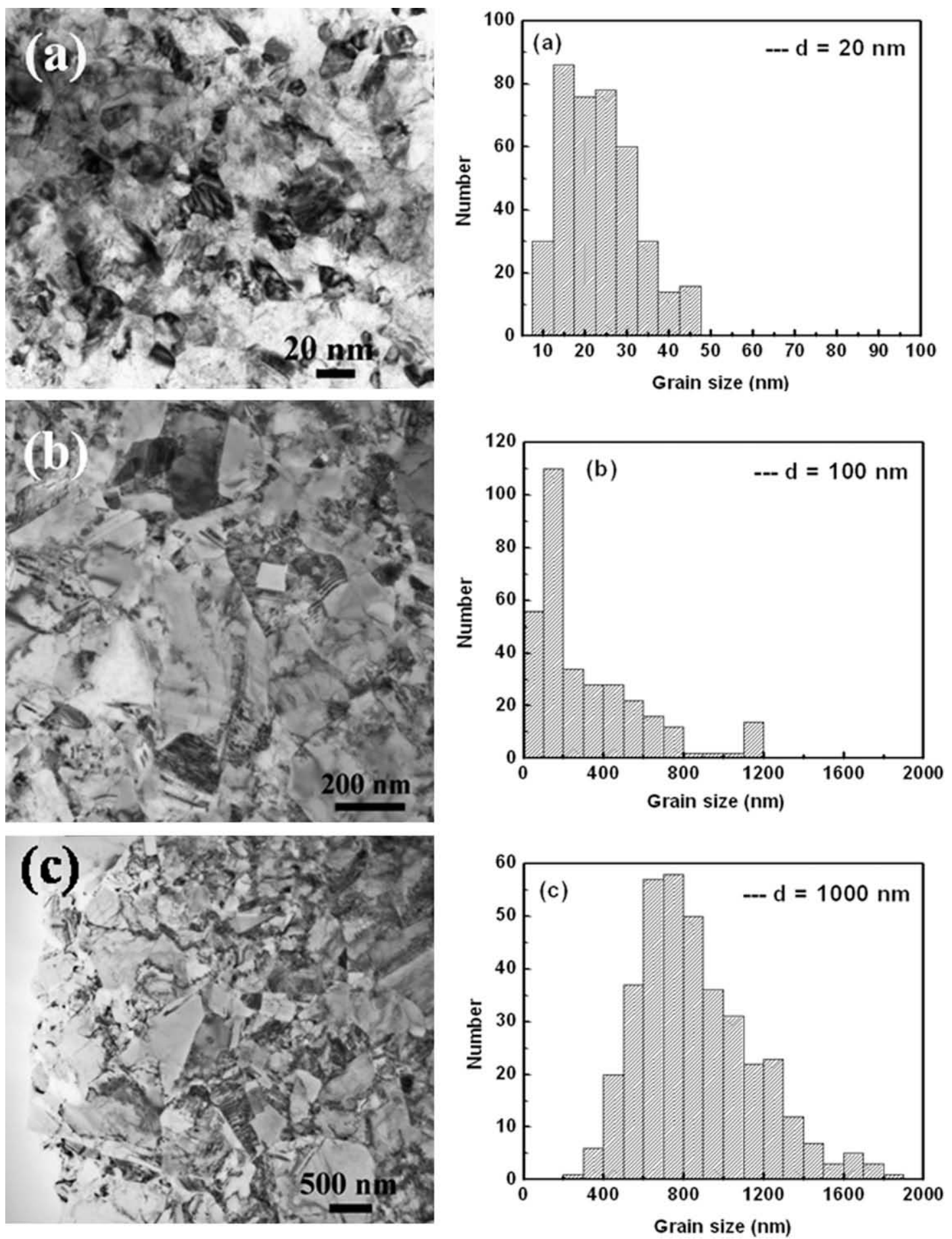

Fig. 1. The microstructures and the grain size histograms of the as-received electrodeposited Ni samples: (a) nanocrystalline Ni, $d=20 \mathrm{~nm}$; (b) UFG Ni, $d=100 \mathrm{~nm}$; (c) UFG Ni, $d=1000 \mathrm{~nm}$.

refinement $[21,22]$. The distribution of the misorientations in Ni100 is slightly different from that in Ni1000 as a higher ratio of small-angle grain boundaries can be seen in sample Ni1000. As discussed later, this difference can affect the deformation behavior.

The EBSD also indicates a preferred grain orientation formulated in the as-received UFG samples (Ni100 and Ni1000), with [100] direction along the sample thickness (sample normal direction), Fig. 3. Although the texture is not strong, it is typical of the growth texture by electrodeposition, which is also similar to that of other as-deposited $\mathrm{Ni}[26,29]$. Nevertheless, nearly random texture was exhibited in the transverse direction (TD in the figure) and longitudinal direction (RD in the figure).

TEM observations did not reveal growth twins or stacking faults in the as-received $\mathrm{Ni}$ samples, but detailed TEM 

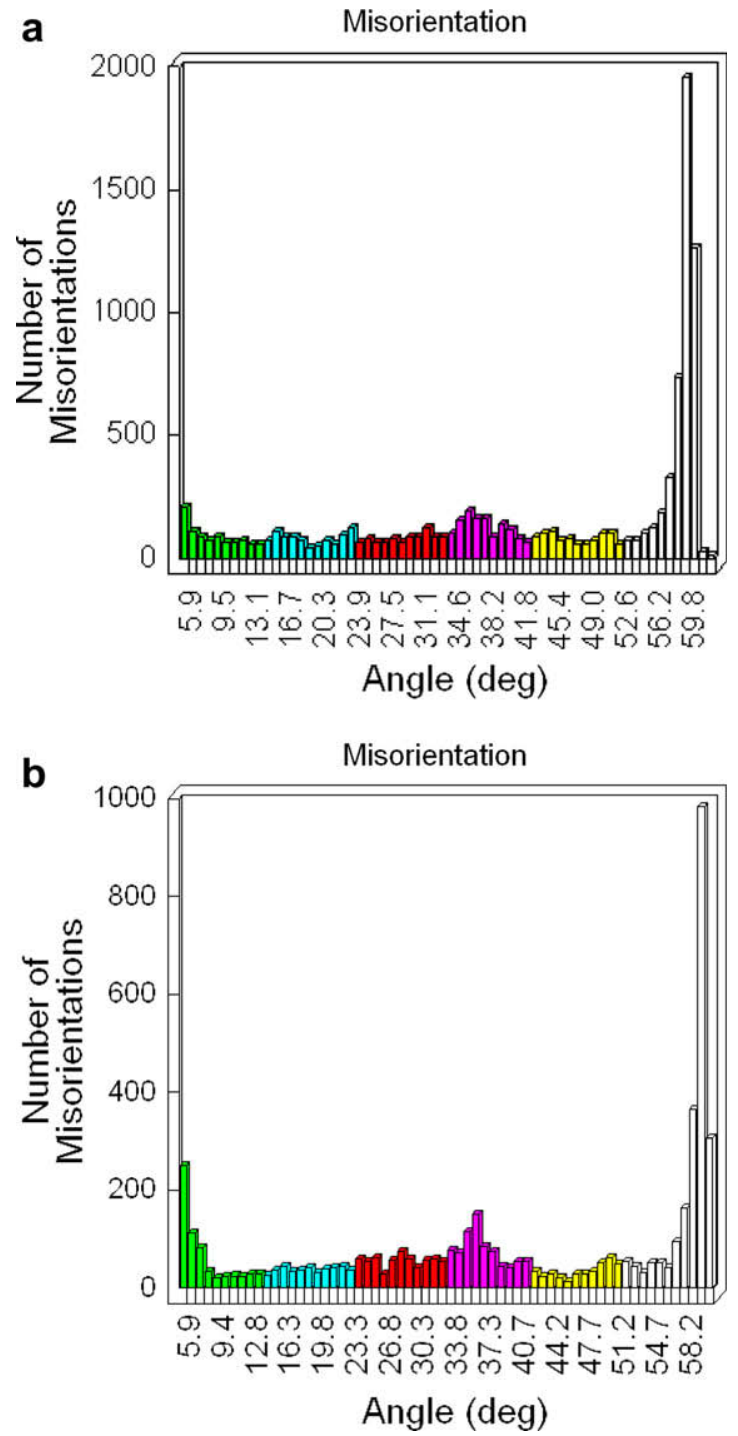

Fig. 2. The grain-misorientation distribution of the as-received UFG Ni: (a) $d=100 \mathrm{~nm}$; (b) $d=1000 \mathrm{~nm}$. Both samples are characteristic of largeangle grain boundaries, but a slightly higher ratio of small-angle grain boundaries $\left(<10^{\circ}\right)$ was in UFG Ni with grain size of $1000 \mathrm{~nm}$.

observations indicate that dislocation clusters with high density were present in Ni1000. Fig. 4 shows the representative microstructure. As pointed by arrows, the dislocation clusters were exhibited in both the grain interior (A) and the grain boundaries (B). These grain boundaries with dislocation clusters appear rough, as shown in Fig. 4, which resemble the small-angle grain boundaries often seen in metals produced by SPD [30]. This observation is actually consistent with the increased ratio of small-angle grain boundaries measured by EBSD (Fig. 2b).

Fig. 5a summarizes the macroscopic strain evolutions of Ni samples with different grain sizes during cyclic deformation, in which the evolutions under $\sigma_{\max }$ levels (represented by solid symbols) and $\sigma_{\min }$ levels (by open symbols) are both shown as a function of the cycle numbers. For all the samples, the macroscopic strains at $\sigma_{\min }$ levels show similar evolution to those measured at $\sigma_{\max }$ levels. But
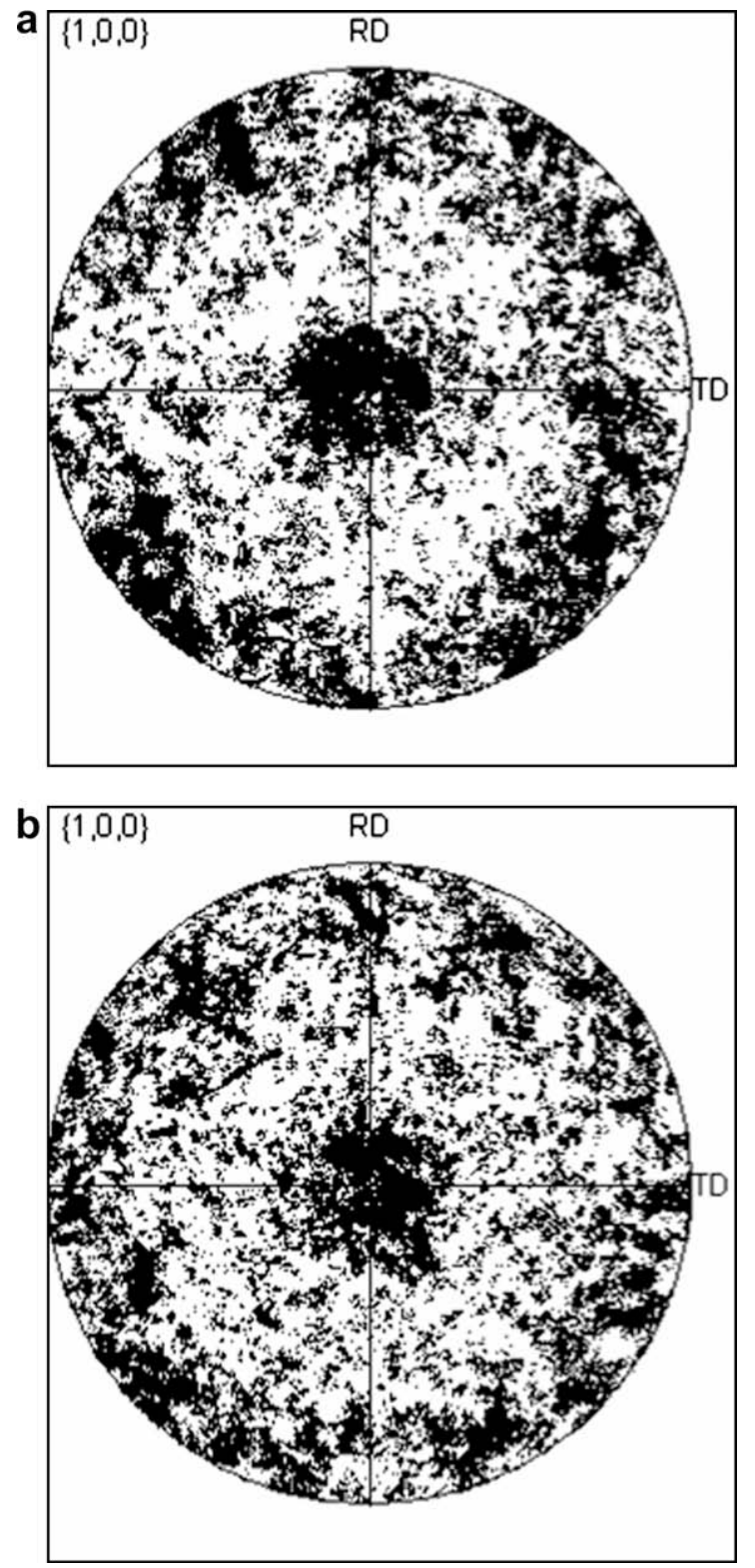

Fig. 3. The texture of the as-received UFG Ni samples: (a) $d=100 \mathrm{~nm}$; (b) $d=1000 \mathrm{~nm}$. The as-received samples exhibit a [100] growth texture in the thickness direction (sample normal is in the center of the figures).

different behavior can be seen for samples with different grain sizes. For the $\mathrm{CG} \mathrm{Ni}$, the macroscopic strains stayed nearly constant with the cycle numbers, whereas for Ni100, the macroscopic strain showed an increase in the first a few cycles, then a slight decrease with the increase of cycle numbers. Two cyclic loading schemes were performed for Ni1000 (one at $\sigma_{\max }=460 \mathrm{MPa}$ and the other at $\sigma_{\max }=560 \mathrm{MPa}$ ). One can see that, at $\sigma_{\max }=460 \mathrm{MPa}$, the macroscopic strain evolution is similar to that of sample Ni100, i.e., the strain slightly increased in the first a few cycles, then slightly decreased with the increase of cycle numbers. For $\sigma_{\max }=560 \mathrm{MPa}$, however, the macroscopic strain showed a monotonic increase with the cyclic numbers (from $055 \%$ to $0.65 \%$ ). Since the cyclic loading was performed under constant load-controlled mode, the 


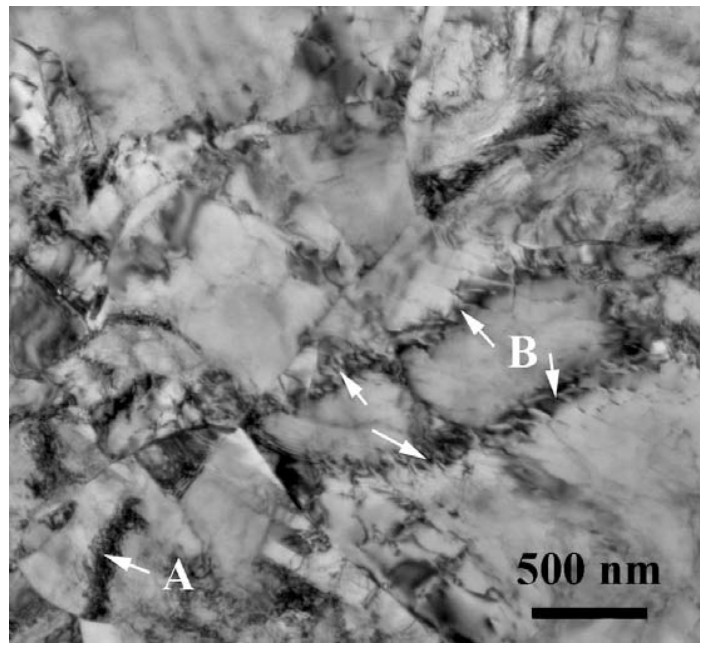

Fig. 4. Bright-field TEM micrograph of as-received UFG Ni $(d=1000 \mathrm{~nm})$. Heavy dislocation clusters are present in both the grains (area marked by A) and at grain boundaries (area marked by B).

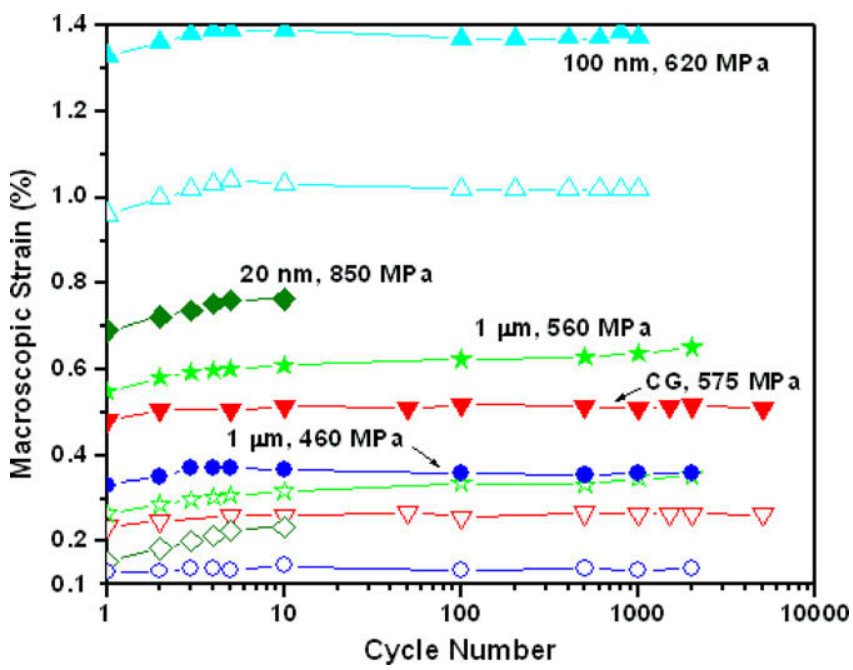

Fig. 5. The macroscopic strain evolution with the cycle numbers, measured during the in situ cyclic deformation. The solid symbols represent the strains at $\sigma_{\max }$, and the open symbols for the strains at $\sigma_{\min }$ that is $50 \mathrm{MPa}$ for all the samples.

increase of macroscopic strain actually indicates sample softening, while a decrease of strain means sample hardening. For the $\mathrm{NC} \mathrm{Ni}$, a slight increase of macroscopic strain was also seen at the beginning, but the full evolution spectrum was not available, as the sample failed at a relatively small cycle number. Thus, the NC Ni is used as comparison, and this study is focused on the UFG samples.

The different cyclic deformation behavior of Ni samples is also reflected in the lattice strain evolution. It is known that in face-centered cubic (fcc) metals, the (200) plane, as the most compliant, usually shows the largest lattice strain upon deformation [31]. Fig. 6 shows the residual lattice strains from (200) reflection (which were all measured at $\sigma_{\min }=50 \mathrm{MPa}$ ). For $\mathrm{NC} \mathrm{Ni}$, the residual strains in the loading direction (LD) and in the transverse direction (TD) were very small and both close to zero, considering the large error bars. For Ni100, the residual lattice strains in LD showed negative values (compressive), while these in TD were positive (tensile), Fig. 6b. Fig. 6c shows the lattice strains of sample Ni1000 in LD and TD at $\sigma_{\max }=460 \mathrm{MPa}$. Similar to that observed in Ni100, the lattice strains in TD were of tensile type, while the lattice strains in LD remained virtually zero. When $\sigma_{\max }$ was increased to $560 \mathrm{MPa}$, higher tensile lattice strains were observed in TD, but the lattice strains in LD began to shift toward negative. We also plotted the residual lattice strain evolution of coarse-grained $(\mathrm{CG}) \mathrm{Ni}$ (also measured at $50 \mathrm{MPa}$ ) for comparison. As shown in Fig. $6 e$, it is in striking contrast that the residual lattice strains in $\mathrm{CG} \mathrm{Ni}$ were both positive (tensile) in LD and $\mathrm{TD}$, with a higher value in LD than in TD. It is worth noting that the same layout of $(200)$ residual lattice strain was observed in a CG Ni alloy in a recent low-cycle fatigue experiment [32]. The scenario exhibited in our CG Ni is also consistent with the observations in other CG metals, which was confirmed as the typical behavior induced by dislocation activities [31,33]. It is clear that the behavior of the (200) lattice strains of UFG Ni is very different from that of CG Ni, indicating that the deformation of UFG Ni under cyclic loading was significantly different.

Our TEM observation indicates that the sample microstructure was not changed after deformation in the $\mathrm{NC}$ $\mathrm{Ni}$, due to the limited cyclic numbers as well as the low applied strains. This is in agreement with the observation by $\mathrm{Wu}$ et al. [34]. By contrast, significant deformation activities were observed in the UFG Ni samples. In general, four types of deformation pathways can be identified under the given deformation conditions: (1) deformation twining; (2) dislocation emitting; (3) stacking fault; and (4) dislocation disentangling. As discussed below, these deformation features help to understand both the macroscopic and lattice strain evolutions observed in the UFG Ni samples.

First of all, in Ni100, the deformation was mainly mediated by deformation twinning and dislocation emitting. Because of the presence of large grains in this sample, the deformation was found to take place preferentially in these large grains. Fig. 7 demonstrates the representative TEM micrographs of Ni100 after the cyclic deformation. In Fig. 7a, the twins were nucleated at the grain boundaries and traversed the grain, whereas the dislocation activity was not very obvious. Due to the high stress required to nucleate intragranular dislocations in UFG metals, they can be alternatively generated from grain boundary sources [35]. As shown in Fig. 7b, the dislocations, activated in the triple junction, were emitted into the grain (shown by an arrow), while no twinning can be observed. Fig. 7c shows both the dislocations and twinning, with the growth of twinning being parallel to the dislocation emission from the grain boundary. In other grains, the dislocation emission can be perpendicular to the twinning length direction, see Fig. 7d; as a result, the dislocations were interwoven by these twin faults. These different morphologies may be relevant to the local stress state enforced by the cyclic loading in peculiarly oriented grains. 

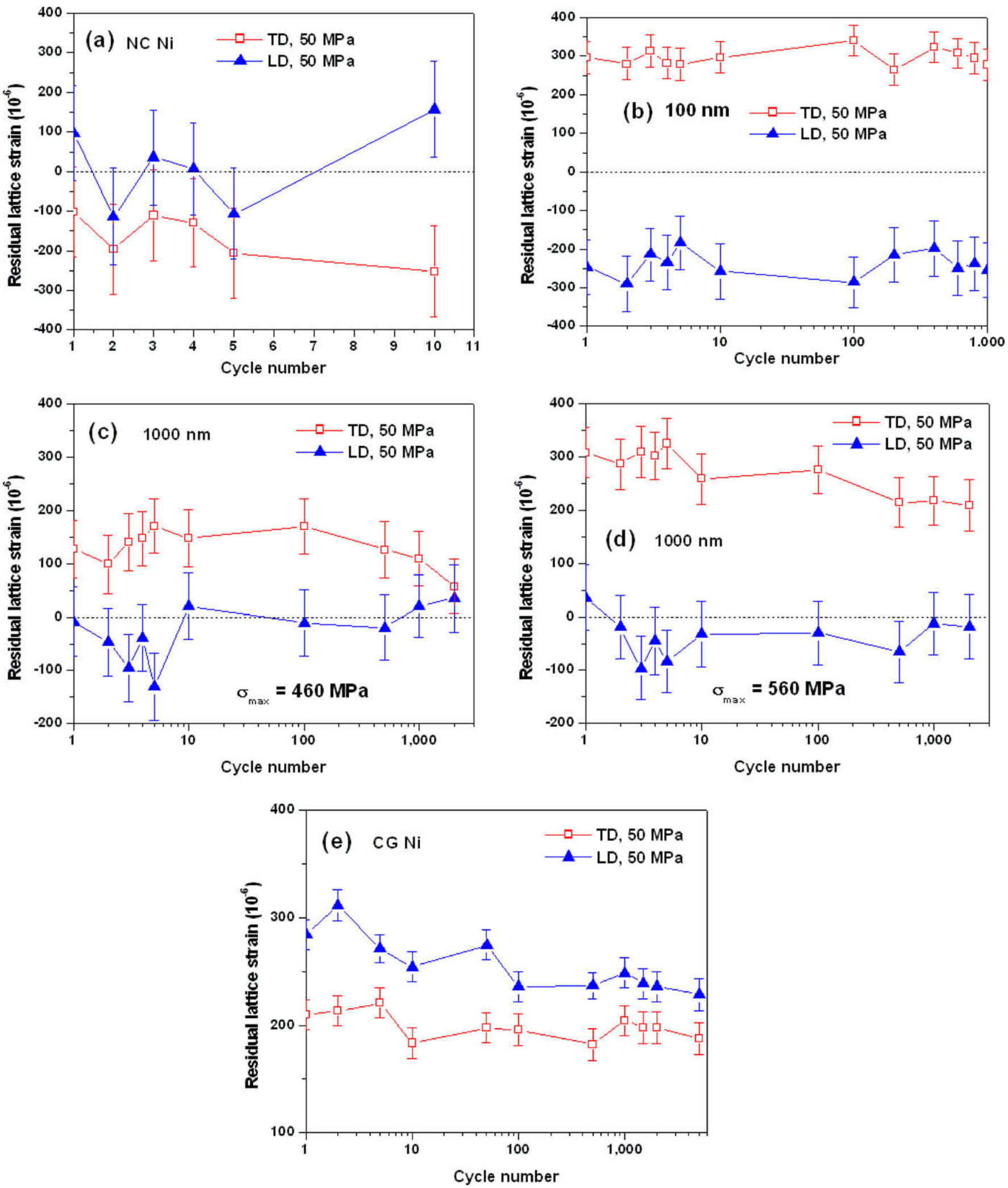

Fig. 6. The evolution of (200) residual lattice strains as a function of cycle numbers in Ni samples with different grain sizes: (a) NC Ni; (b) $d=100 \mathrm{~nm}$; (c) $d=1000 \mathrm{~nm}, \sigma_{\max }=460 \mathrm{MPa}$; (d) $d=1000 \mathrm{~nm}, \sigma_{\max }=560 \mathrm{MPa}$; (e) CG Ni. The residual lattice strains were all measured at $\sigma_{\min }=50 \mathrm{MPa}$.

The unusual residual lattice strains observed in LD is believed to be a result of the deformation twinning. As twinning was not observed in our CG Ni (which is consistent with other studies [36]), the obvious difference in lattice strains between the UFG Ni and the CG Ni indicates that deformation twinning dramatically altered the lattice strains between 

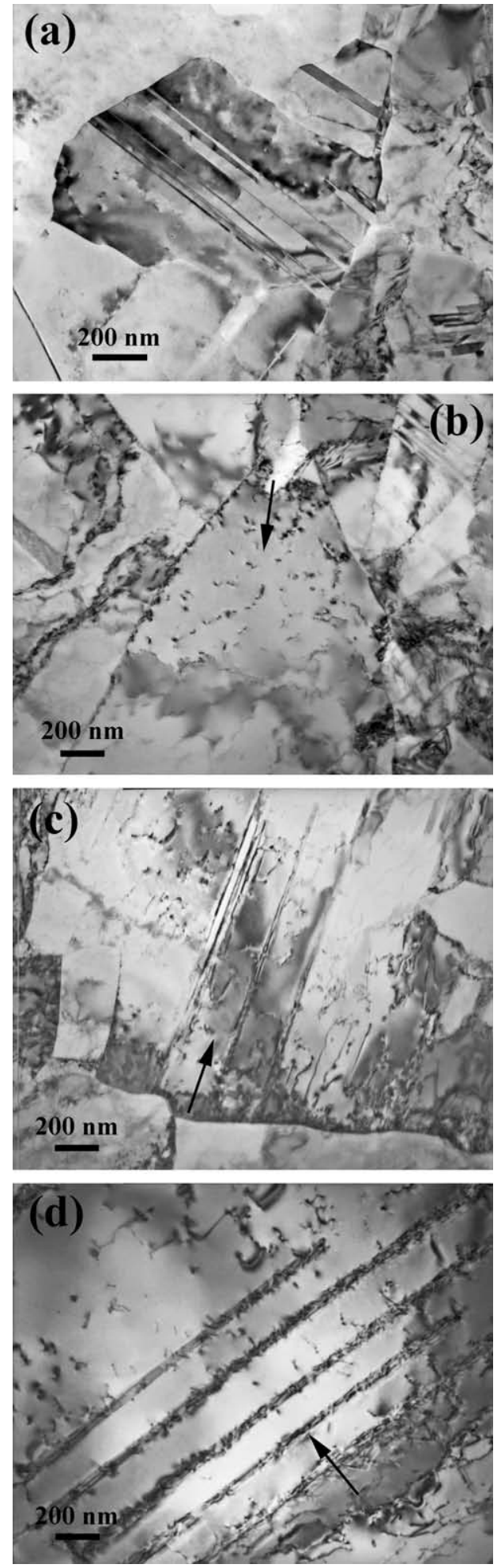

Fig. 7. Representative bright-field TEM micrographs of UFG Ni $(d=100$ $\mathrm{nm}$ ) after cyclic loading. (a) The deformation twins were nucleated from the grain boundary and traversed the grain. (b) The dislocations were emitted from the grain boundary source. (c) The dislocation emission was in parallel to the twin length. (d) The dislocation emission was right to the twin length, and they were entangled by the twins.

the LD and TD in the UFG Ni. Furthermore, since the unusual lattice strains were formulated in the first loading cycle, it suggests that deformation twinning occurred in the first stroke on the specimen, which is virtually a monotonic loading. Indeed, it was verified in our uniaxial tensile loading experiments that deformation twinning contributed to the unusual residual lattice strains in the LD; in addition, the extent of twinning (both the twin size and density) was scaled with the deformation amount [12]. For sample Ni100, as a higher macroscopic strain was reached $\left(1.33 \%\right.$ at $\left.\sigma_{\max }\right)$ in the first cycle, the higher twinning activity induced the large reversal of lattice strains in LD and TD. For sample Ni1000, smaller macroscopic strains were enforced during the first loading cycle, e.g., $\sim 0.35 \%$ at $\sigma_{\max }=460 \mathrm{MPa}$ and $\sim 0.55 \%$ at $\sigma_{\max }=560 \mathrm{MPa}$, respectively (see Fig. 5). Therefore, a less displacement of lattice strain toward negative in LD is seen than that in $\mathrm{Ni}$ with grain size of $100 \mathrm{~nm}$. After the first cycle, little growth of twinning was found under load-controlled cyclic loading; the deformation thereafter is mainly mediated by dislocation activities, which are accounted for the macroscopic strain evolution. As mentioned earlier, in the initial cyclic loading (up to 10 cycles or so), the sample exhibited a softening, which may be related to the resistance to the dislocation emission. When the dislocation emission was just activated from the boundary sources, there were relatively few obstacles present in their paths; the dislocations could thus glide readily in the grain. After the emitted dislocations started to accumulate, as shown in Fig. 7, the dislocation density was increased, and the sample hardening emerged. Moreover, the interaction of the dislocations with twins may be the other factor that could greatly contribute to the hardening.

For sample Ni1000, at $\sigma_{\max }=460 \mathrm{MPa}$, both twinning and dislocation activities were found in the samples after deformation, although their density was far less than what was observed in sample Ni100. Fig. 8a shows a representative observation, in which thin deformation twins were generated in a grain (pointed by arrows in the figure) while its neighbors were almost twin-free. In addition, due to the small applied strains, the dislocation activities were also less intense than in Ni100. But the discontinuous dislocations accumulated in the grain after the initial cycling, as shown in Fig. 8b. Although the interaction of dislocations with twins was not commonly seen in the sample, it is believed that the dislocation accumulation is responsible for the material hardening, Fig. 5b.

By contrast, the deformation at $\sigma_{\max }=560 \mathrm{MPa}$ showed more pronounced dislocation and twinning activities. Fig. 9a shows the representative TEM observations. One can see that a number of dislocations were generated from the grain boundaries and traveled deep into the grain interior (as pointed by the black arrow); the dislocations also began interweaving with the twins. It is of interest to note that the dislocation emissions were exclusively from large-angle grain boundaries in both Ni100 and Ni1000. Moreover, as compared to that at $\sigma_{\max }=460 \mathrm{MPa}$, the twinning activities were more intense as both the twin width and length increased. The enhanced twinning activities are believed to shift the lattice strain in LD toward the negative, Fig. 6d. 

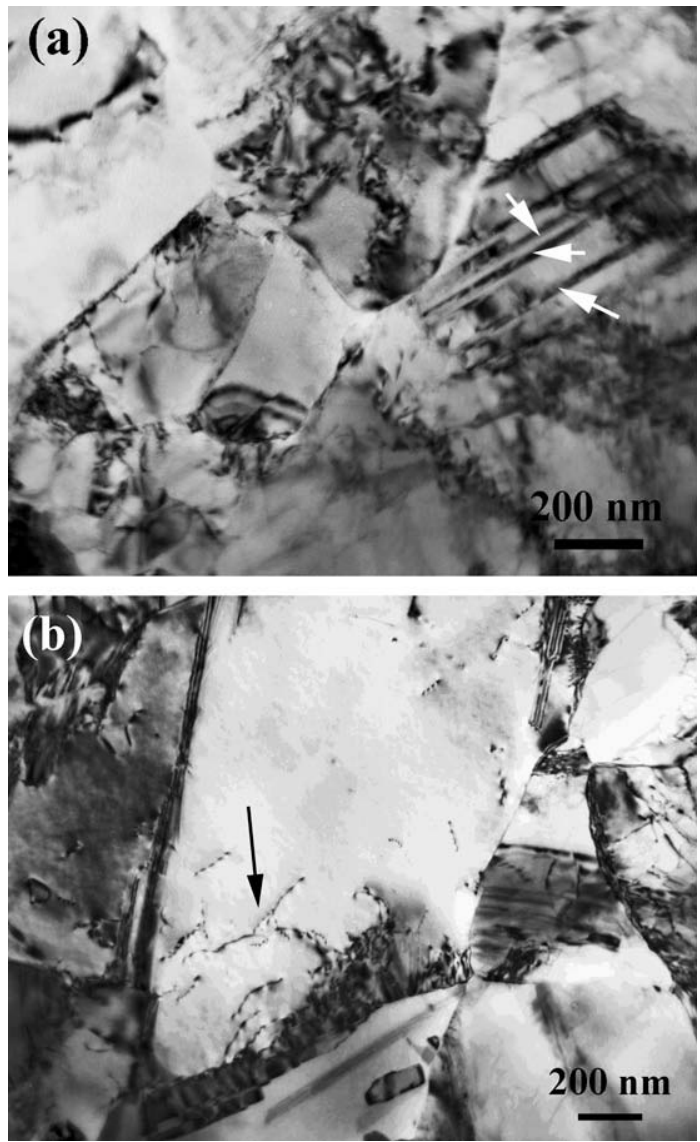

Fig. 8. Representative bright-field TEM micrographs of UFG Ni $(d=1000 \mathrm{~nm})$ after cyclic loading at $\sigma_{\max }=460 \mathrm{MPa}$. (a) Deformation twins formed in some grains, but with smaller density due to the small applied strain. (b) Dislocations emitted from the grain boundary source, and accumulated in the grain.

Other than the twinning, stacking faults were also observed under this loading scheme. Fig. 9b shows the interaction of dislocations with the stacking faults. Despite the stronger dislocation accumulation and interaction with twins and stacking faults, this sample exhibited apparent softening, Fig. 5 b. With $\sigma_{\max }=560 \mathrm{MPa}$, it is found that heavy dislocation clusters that were observed at both the grain interiors and grain boundaries in the as-received samples (area $\mathrm{A}$ and B in Fig. 4) unraveled after cyclic deformation, Fig. 9c and d, which likely leads to dislocation annihilation and an overall decrease in the dislocation density. As a matter of fact, the variation of dislocation density was confirmed by the peak width measurement during the in situ neutron diffraction. Fig. 10 shows the evolution of the full width at half maximum (FWHM) of (1 111 ) peak at $\sigma_{\max }=560 \mathrm{MPa}$. It is clear that the peak width increases in the first a few cycles, and then gradually decreases with the cycle numbers. The peak width variation attests the overall change of dislocation density, as revealed by TEM observations. Since the strength of the materials is related to the dislocation density by $\sigma_{f} \propto \rho^{0.5}$. As a result, the macroscopic sample softening was shown. At $\sigma_{\max }=460 \mathrm{MPa}$, due to the lack of dislocation unraveling mechanism, the softening behavior was absent.
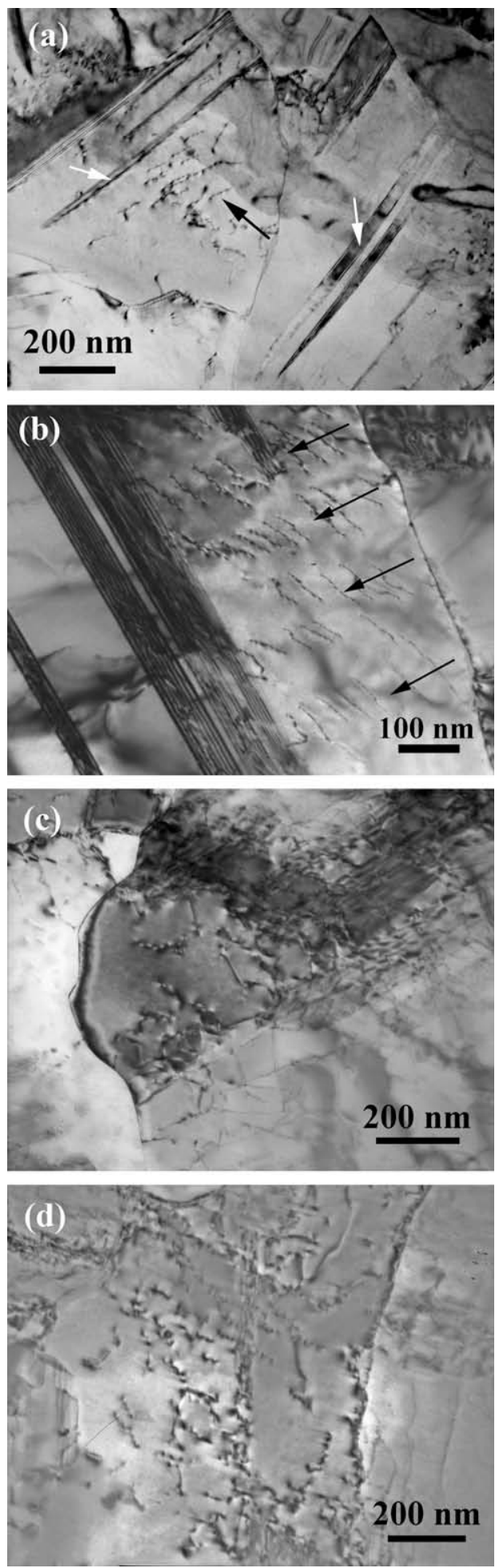

Fig. 9. Representative TEM micrographs of UFG Ni $(d=1000 \mathrm{~nm})$ after cyclic loading at $\sigma_{\max }=560 \mathrm{MPa}$. (a) Dislocations emitted from the grain boundary source together with the deformation twins generated in the same grain. (b) Stacking faults formed during deformation, and the dislocation emission interacted with the stacking faults. (c) and (d) Dislocation tangles separated after cyclic deformation.

Strong cyclic softening was previously observed in UFG $\mathrm{Cu}$ produced by ECAP, which was attributed to the general decrease of the defect density during cyclic tests $[24,37,38]$. 


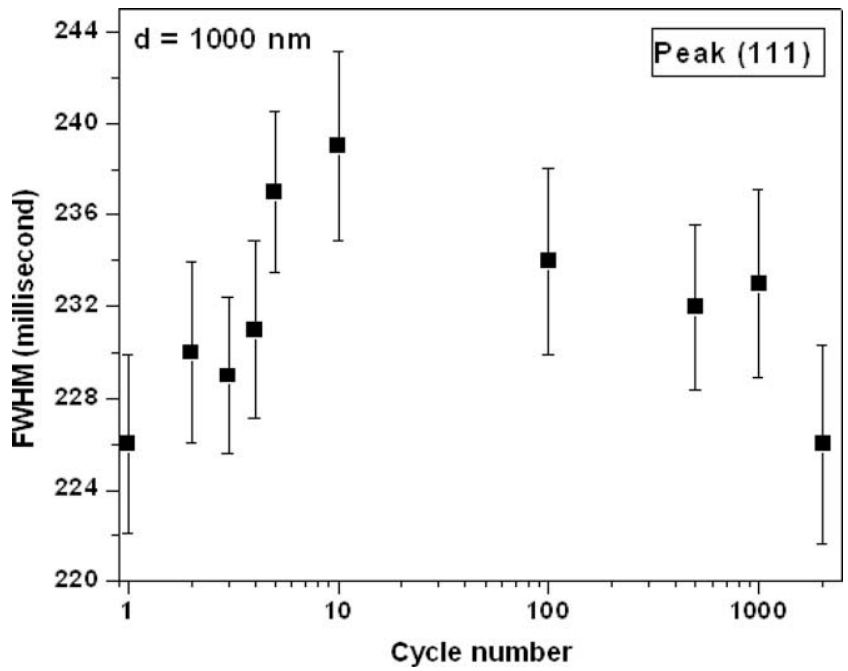

Fig. 10. The FWHM evolution of (1 111$)$ peak of UFG Ni $(d=1000 \mathrm{~nm})$ during cyclic deformation at $\sigma_{\max }=560 \mathrm{MPa}$. The peak width was measured during the in situ time-of-flight neutron diffraction.

In common, those ECAP-processed samples are characterized by small-angle grain boundaries, and the grains are in a sense similar to the subgrains or the dislocation cells. The unstable grain boundaries can be activated and rearranged into more stable structure under the drive of cyclic deformation [24]. In our UFG Ni, even though there is only a small ratio of small-angle grain boundaries, their contribution to the deformation behavior can be influential when the applied stress is increased to a certain level (e.g., $\sigma_{\max }=560 \mathrm{MPa}$ here).

\section{Summary}

In summary, UFG Ni samples made by electrodeposition were characterized by large-angle grain boundaries. In situ neutron diffraction indicates unusual residual lattice strains. Cyclic hardening/softening was observed in these UFG samples under different loading conditions. The dislocation emission and accumulation from grain boundaries were responsible for the cyclic hardening, while dislocation disentangling led to a decrease of dislocation density, which was accounted for the cyclic softening.

\section{Acknowledgements}

Financial support was through the NSF Major Research Instrumentation Program (DMR-0421219) and NSF International Materials Institutes program (DMR0231320). XLW, ADS and JAH acknowledge support by Division of Materials Sciences and Engineering, Office of Basic Energy Sciences, US Department of Energy under Contract DE-AC05-00OR22725 with UT-Battelle, LLC. The neutron diffraction was conducted at the Lujan Neutron Scattering Center at LANSCE funded by the Depart- ment of Energy's Office of Basic Energy Science. The Los Alamos National Laboratory (LANL) is operated by the Los Alamos National Security LLC under the DOE Contract DEAC52-06NA25396.

\section{References}

[1] Zhang K, Weertman JR, Eastman JA. Appl Phys Lett 2005;87:061921.

[2] Youssef KM, Scattergood RO, Murty KL, Horton JA, Koch CC. Appl Phys Lett 2005;87:091904.

[3] Kumar KS, Van Swygenhoven H, Suresh H. Acta Mater 2003;51:5743.

[4] Gianola DS, Van Petegem S, Legros M, Brandstetter S, Van Swygenhogen H, Hemker KJ. Acta Mater 2006;54:2253.

[5] Wang YM, Hamza AV, Ma E. Acta Mater 2006;54:2715.

[6] Zhu YT, Liao XZ, Valiev RZ. Appl Phys Lett 2005;86:103112.

[7] Shan Z, Wiezorek JMK, Stach EA, Follstaedt DM, Knapp JA, Mao SZ. Phys Rev Lett 2007;98:095502.

[8] Wu XL, Qi Y, Zhu YT. Appl Phys Lett 2007;90:221911.

[9] Wu XL, Ma E, Zhu YT. J Mater Sci 2007;42:1427.

[10] Budrovic Z, Van Swygenhoven H, Derlet PM, Van Petegem S, Schmitt B. Science 2004;304:273.

[11] Budrovic Z, Van Petegem S, Derlet PM, Schmitt B, Van Swygenhoven $\mathrm{H}$, Schafler E, et al. Appl Phys Lett 2005;86:231910.

[12] Cheng S, Stoica AD, Wang XL, Ren Y, Almer J, Horton JA, et al. Phys Rev Lett, submitted for publication.

[13] Fan GJ, Choo H, Liaw PK. Appl Phys Lett 2006;89:061919.

[14] Farkas D, Willemann M, Hyde B. Phys Rev Lett 2005;94:165502.

[15] Yang Y, Imasogie B, Fan GJ, Liaw PK, Soboyejo WO. Metall Mater Trans A 2008;39:1145.

[16] Hanlon T, Tabachnikova ED, Suresh S. Intl J Fatigue 2005;27:1147.

[17] Moser B, Hanlon T, Kumar KS, Suresh S. Scripta Mater 2006;54:1151.

[18] Cheng S, Stoica AD, Wang X-L, Wang GY, Choo H, Liaw PK. Scripta Mater 2007;57:217.

[19] Huebner P, Kiessling R, Biermann H, Hinkel T, Jungnickel W, Kawalla R, et al. Metall Mater Trans A 2007;38:1926.

[20] Hanlon T, Kwon Y-N, Suresh S. Scripta Mater 2003;49:675.

[21] El-Danaf EA. Mater Sci Eng A 2008;487:189.

[22] Cheng S, Zhao YH, Zhu YT, Ma E. Acta Mater 2007;55:5822.

[23] Chen Y, Li Y, He L, Lu C, Ding H, Li Q. Mater Lett 2008;62:2821.

[24] Agnew SR, Weertman JR. Mater Sci Eng A 1998;244:145.

[25] Höppel HW, Kautz M, Xu C, Murashkin M, Langdon TG, Valiev RZ, et al. Int J Fatigue 2006;28:1001.

[26] Batane NR, Morrison DJ, Moosbrugger JC. Scripta Mater 2008;58:955

[27] Baker I, Li J. Microsc Res Tech 2004;63:289.

[28] Gottstein G, Shvindlerman LS. Grain boundary migration in metals: thermodynamics, kinetics, applications. Berlin: CRC Press; 1999. p. 110.

[29] Kulovits A, Mao SX, Wiezorek JMK. Acta Mater 2008;56:4836.

[30] Huang JY, Zhu YT, Jiang HG, Lowe TC. Acta Mater 2001;49:1497.

[31] Clausen B, Lorentzen T, Leffers T. Acta Mater 1998;46:3087.

[32] Huang EW, Clausen B, Wang YD, Choo H, Liaw PK, Benson ML, et al. Int J Fatigue 2007;29:1812.

[33] Wang YD, Tian H, Stoica AD, Wang XL, Liaw PK, Richardson JW. Nature Mater 2003;2:101

[34] Wu XL, Zhu YT, Ma E. Appl Phys Lett 2006;88:121905.

[35] Cheng S, Spencer JA, Milligan WW. Acta Mater 2003;51:4505.

[36] Pang JWL, Rogge RB, Donaberger RL. Mater Sci Eng A 2006;437:21.

[37] Höppel HW, Zhou ZM, Mughrabi H, Valiev RZ. Phil Mag A 2002;8:1781.

[38] Niendorf T, Canadinc D, Maier HJ, Karaman I. Metall Mater Trans A 2007;38:1946. 